\title{
Enhancing Phase-Margin of OTA Using SelF- Biasing CASCODE CURRENT MirRor
}

\author{
Meysam Akbari ${ }^{1}$, Masoud Nazari ${ }^{2}$ and Ardavan Javid ${ }^{3}$ \\ ${ }^{1,2}$ Microelectronic laboratory, Shahid Beheshti University, G. C., Tehran, Iran \\ ${ }^{3}$ ICAS laboratory, K.N. Toosi University of Technology, Tehran, Iran.
}

\begin{abstract}
In this paper, a new adaptive biased low voltage cascode current mirror with high input/output swing is presented. This advantage is achieved using a self-biasing transistor and compensation resistor. The new structure profits from better input dynamic range and lower supply voltage without frequency response limitation and increasing input impedance. Also, the proposed current mirror is incorporated in folded cascode amplifier in order to enhance its phase-margin. The simulation results in $0.18 \mu m$ CMOS technology confirm the theoretical analysis and exhibits $478 \mu \mathrm{A}$ linear input/output current swing and a phase-margin enhancement of $12^{\circ}$ for the proposed current mirror and amplifier compared to the conventional circuits, respectively.
\end{abstract}

\section{KEYWORDS}

Swing, Current mirror, Dynamic range, Folded cascade, Phase-margin, Self-biasing.

\section{INTRODUCTION}

The demand for low power circuits is increased in last decades. The portable devices are the main purpose of these circuits due to limited power supply availability. The current mirror $(\mathrm{CM})$ is one of the main parts of the most analogue and mixed-signal integrated circuits such as OTAs, current conveyers and current sensors. The low voltage cascode current mirror (CCM) has high swing and small input voltage compared to the conventional CCM. These properties make it ideal for VLSI test circuits and portable devices [1]. A low voltage CCM is shown in Fig. 1. The bias voltage $V_{b}$ should be set according to the input current $I_{i n}$ in order to ensure saturation operation of all transistors. By increasing input current the operation of transistor M1 may be changed into triode region. This limitation is imposed by fixed bias voltage $V_{b}$ which can't increase proportionally with input current [2]. Recent works on the CM include biasing resistor [3], linearregion transistor [3] and level shifter transistor [4] to increase input dynamic range and introduce bias voltage $V_{b}$ of the low voltage CCM. But these topologies lead to the higher input voltage requirement, increasing input impedance and silicon area compared to the conventional $\mathrm{CM}$. For example, a self-biasing low voltage CCM can be used in the operational transconductance amplifiers (OTAs) as the transconductance conveyer structure [5]. But this configuration leads to the phase-margin (PM) degeneration due to the larger parasitic capacitances of the self-biasing topology [6]. Therefore, the high speed CM can be introduced to compensate of such phasemargin degeneration $[7,8]$.

In this paper, a new self-biasing CCM with frequency compensation technique is introduced. It can operate well in low supply voltage and it has high input/output dynamic range. Also, it can be used in folded cascode (FC) amplifier in order to phase margin enhancement. The paper is organized as follow: Section 2 introduces proposed CCM with a brief comparison to the recent

DOI : 10.14810/elelij.2014.3403 
works. In the next section, the enhanced phase-margin single ended FC amplifier is described. The simulation results are discussed in Section 4 followed by a conclusion in Section 5 .

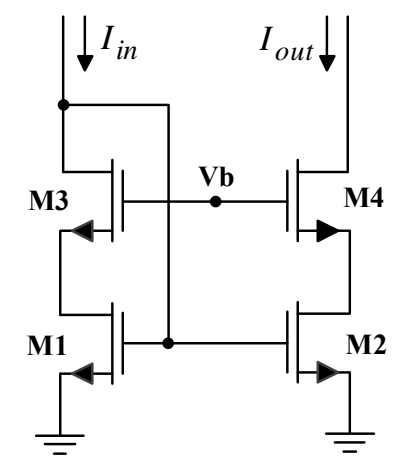

Figure 1. Conventional low voltage cascode current mirror.

\section{Proposed Current Mirror (PCM)}

As shown in Fig. 2a, a resistor in series with input node can be used to produce bias voltage of M1 and M3 [3]. Despite the simplicity and increased input current range of this architecture, the integrated resistor suffers from process variation, so the voltage of the gate terminals will not be precise. In addition, the value of the resistor should be decreased as input current rise further. Another architecture of the pervious circuit is shown in Fig. 2b. A linear-region MOS transistor as resistor is used in this structure which its gate is connected to the input and value of the resistor can be changed by input current [3]. Although the problems of current mirror in Fig. 2a are diminished, but minimum input voltage is approximately twice the value needed in the conventional low voltage CCM. Thus, architecture of Fig. $2 \mathrm{~b}$ is not suitable for low voltage circuits. In addition, the input resistor of CM is increased which is not desirable for CCM. A level shifted current mirror can be used in conventional low voltage CCM for another approach [4]. As shown in Fig. 2c, when $I_{\text {in }}$ increases ( $V_{\text {in }}$ increases too), the transistor M5 shifts the voltage level at the gate terminal of M3. As a result, input current can be increased more than conventional $\mathrm{CM}$. Therefore, the problems of current mirrors in Figs. $2 \mathrm{a}$ and $2 \mathrm{~b}$ are eliminated, but the chip area of current mirror in Fig. $2 \mathrm{c}$ is more than its counterparts. This area arises from the large size of transistor M5 and an additional bias current source. The transistor M5 should operate in subthreshold region to achieve proper performance, so $I_{\text {bias }}$ should be small enough to keep M5 at this region and consequently the aspect ratio of transistor M5 should be large [4].

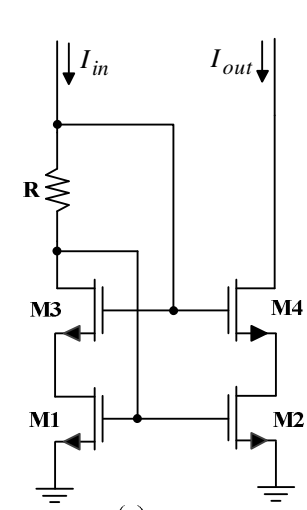

(a)

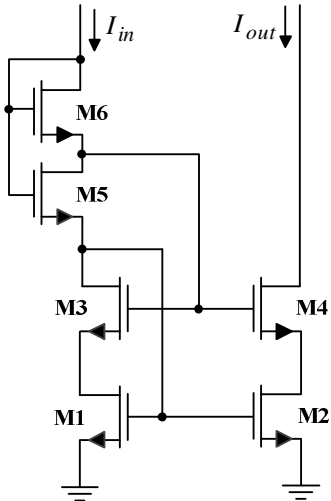

(b)

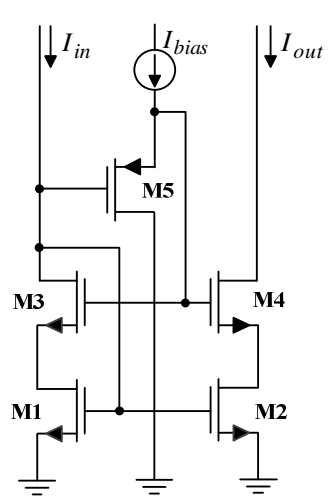

(c)

Figure 2. Cascode current mirrors with adaptive biasing: (a) resistor biasing of cascode transistors, (b) linear-region transistor biasing of cascode transistors, and (c) level shifted current mirror. 
The proposed CM is illustrated in Fig. 3. In this circuit, PMOS transistor M5 creates a bias voltage for cascode transistors M3 and M4 of current mirror. The body effect is removed by connecting the body to the source terminal of M5 for decreasing the input voltage requirement. To ensure saturation operation of M1 and M3, the input voltage $V_{\text {in }}$ should be

$$
V_{i n}=V_{S G 5}+V_{o v 1}=V_{t h}+V_{o v 3}+V_{o v 1}
$$

where $V_{o v 1}$ and $V_{o v 3}$ are overdrive voltage of transistors $\mathrm{M}_{1}$ and $\mathrm{M}_{3}$ which are founded by (2) and (3), respectively.

$$
\begin{gathered}
V_{o v 1}=\sqrt{\frac{2 I_{i n}}{K^{\prime} A_{1}}} \\
V_{o v 3}=\sqrt{\frac{2 I_{i n}}{K^{\prime} A_{3}}}
\end{gathered}
$$

where $A_{1}=W_{1} / L_{1}, A_{3}=W_{3} / L_{3}$ and $W_{j}, L_{j}$ are width and length of transistor $\mathrm{M}_{\mathrm{j}}$, respectively.

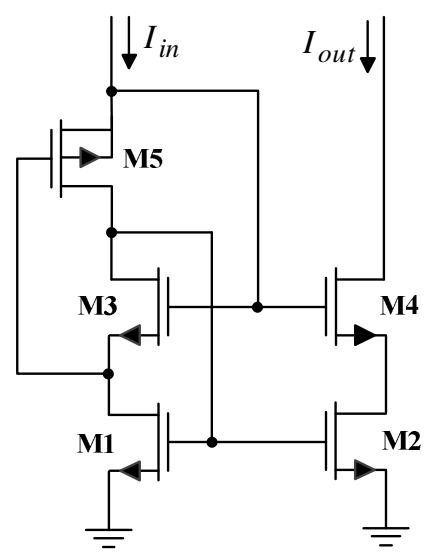

Figure 3. Proposed low volatge cascode current mirror.

The body effect is ignored and it is assumed that all transistors have the identical threshold voltage $V_{t h}$ and transconductance parameter $K^{\prime}=C_{o x} \mu$. The input voltage and input current can be found by replacing equations (2) and (3) in (1).

$$
\begin{gathered}
V_{\text {in }}=V_{t h}+2 V_{\text {od }}=V_{t h}+\sqrt{\left(\frac{2 I_{\text {in }}}{K^{\prime}}\right)\left(\frac{1}{A_{1}}+\frac{1}{A_{3}}\right)} \\
I_{\text {in }, \text { min }}=\frac{K^{\prime}\left(V_{\text {in }}-V_{t h}\right)^{2}}{2\left(\frac{1}{\sqrt{A_{1}}}+\frac{1}{\sqrt{A_{3}}}\right)^{2}}
\end{gathered}
$$

From the above equations, it can be seen there is a relation between input voltage $V_{\text {in }}$ and input current $I_{\text {in }}$ that leads to the self-biasing CCM configuration and a large upper limit. Because all input current/voltage changes will transfer to the cascode transistors through M5. Therefore not only the circuit has a very large upper input current limit $\left(I_{i n, \max }\right)$ but also it has very small value of current gain error rate $\left(I_{\text {out }}-I_{\text {in }}=0\right)$ compared to the conventional CMs. Transistor M5 leads to 
bandwidth degeneration of CCM due to new added parasitic capacitances. But this problem can be resolved by compensation resistor [7, 8]. As shown in Fig. 4 by adding a transistor M6 in deep triode operation region, the new transfer function of circuit can be written by

$$
H(s)=\frac{\frac{g m_{1}}{c_{3}}\left(S+\frac{1}{R_{6} c_{1}}\right)\left(S+\frac{g m_{3}}{c^{\prime}}\right)}{S^{3}+S^{2}\left(\frac{R_{6} c_{1} k g m_{3}+c^{\prime}(k+1)}{R_{6} c^{\prime} c_{1} k}\right)+S\left(\frac{g m_{3}\left(k^{2} c_{3}+k c^{\prime}\right)+k g m_{5} c_{3}}{R_{6} c^{\prime} c_{1} c_{3} k^{2}}\right)+\frac{g m_{3} g m_{5}}{R_{6} c_{1} c_{3} k}}
$$

Where $k$ is the current gain $\left(k=I_{\text {out }} Y_{\text {in }}\right), k C_{1}=C_{g s 2}=k C_{g s 1}, k C_{3}=k C_{g s 3}=C_{g s 4}$ and $C^{\prime}=C_{g s 3}+C_{g s 5}$ are the gate-source capacitances, and $g m_{2}=\mathrm{kgm}_{1}, \mathrm{gm}_{4}=\mathrm{kgm}_{3}$ and $\mathrm{gm}_{5}$ are the transconductance of each transistor and $R_{6}$ is the resistor value of the M6. The transistor M6 generates a pole-zero pair to the transfer function and does not consume power due to the deep triode operation region. The new transfer function has 2 zeroes and 3 poles. By increasing the value of $R_{6}$, the new added polezero pair move from negative infinity toward the origin of the axes. The maximum bandwidth occurs when the zero cancels the low-frequency pole. In this condition, the value of $R_{6}$ can be obtained by

$$
R_{6}=\frac{c^{\prime}(k+1)}{k g m_{3}\left(c_{g s 1}+c_{g d 6}\right)} \approx \frac{c^{\prime}(k+1)}{k g m_{3}\left(c_{g s 1}\right)}
$$

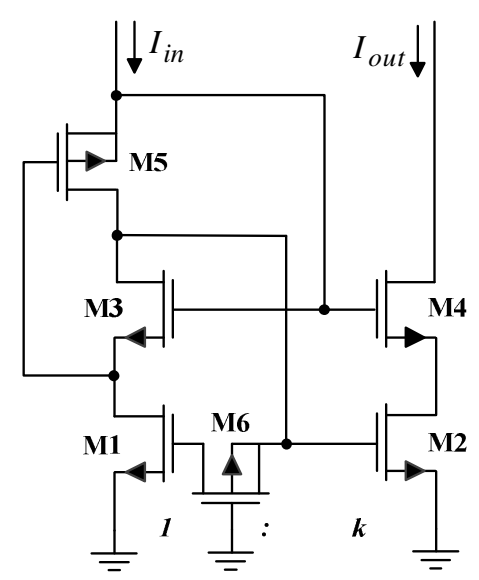

Figure 4. Proposed low volatge cascode current mirror with frequency compensation.

Furthermore, the input impedance is approximately as low as conventional CCM due to the feedback path from gate of M5 to the drain of M1 [9]. The input impedance can be calculated by

$$
R_{\text {in }}=\frac{1}{g m_{1}}\left[1+g m_{1} r_{o 5} \frac{r_{o 5}\left(g m_{3}-g m_{5}\right)}{g m_{5} r_{o 5}+g m_{3} r_{o 3}}\right]
$$

By assuming $g m_{3}=g m_{5},(8)$ can be simplified to

$$
R_{i n}=\frac{1}{g m_{1}}
$$




\section{Enhanced Phase-Margin Folded Cascode Amplifier}

The conventional single ended folded cascode (FC) amplifier in [5] has two prominent poles $\omega_{P 1}$, $\omega_{P 2}$ which are obtained by (10) and (11), respectively.

$$
\begin{gathered}
\omega_{p 1}=-\frac{1}{R_{\text {out }} C_{\text {load }}} \\
\omega_{p 2}=-\frac{g m_{1}}{2 C_{g s 1}}
\end{gathered}
$$

Also, it has a zero at $\omega_{z 1}=2 \omega_{p 2}[5,6]$. In order to have a good phase-margin, $\omega_{p 2}$ can be chosen $\omega_{p 2} \geq 3 \omega_{u}$, where $\omega_{u}$ is unity gain frequency of amplifier. By placing proposed low voltage CCM in folded nodes of the conventional FC topology which is described in [6], the new structure with proposed CCM is shown in Fig. 5.

In this amplifier, the non-dominant pole moves further away from the origin and a new zero added which can be chosen such that cancel the transposed pole. The transfer function of current mirror from ground node to folded node (node X) becomes (M3, M4 excluded)

$$
H(s)=\frac{I_{2}(S)}{I_{1}(S)}=\frac{g m_{2}\left[S+\left(1 / R_{6} C_{g s 1}\right)\right]}{C_{g s 1} S^{2}+\left(2 / R_{6}\right) S+\left(g m_{1} / R_{6} C_{g s 1}\right)}
$$

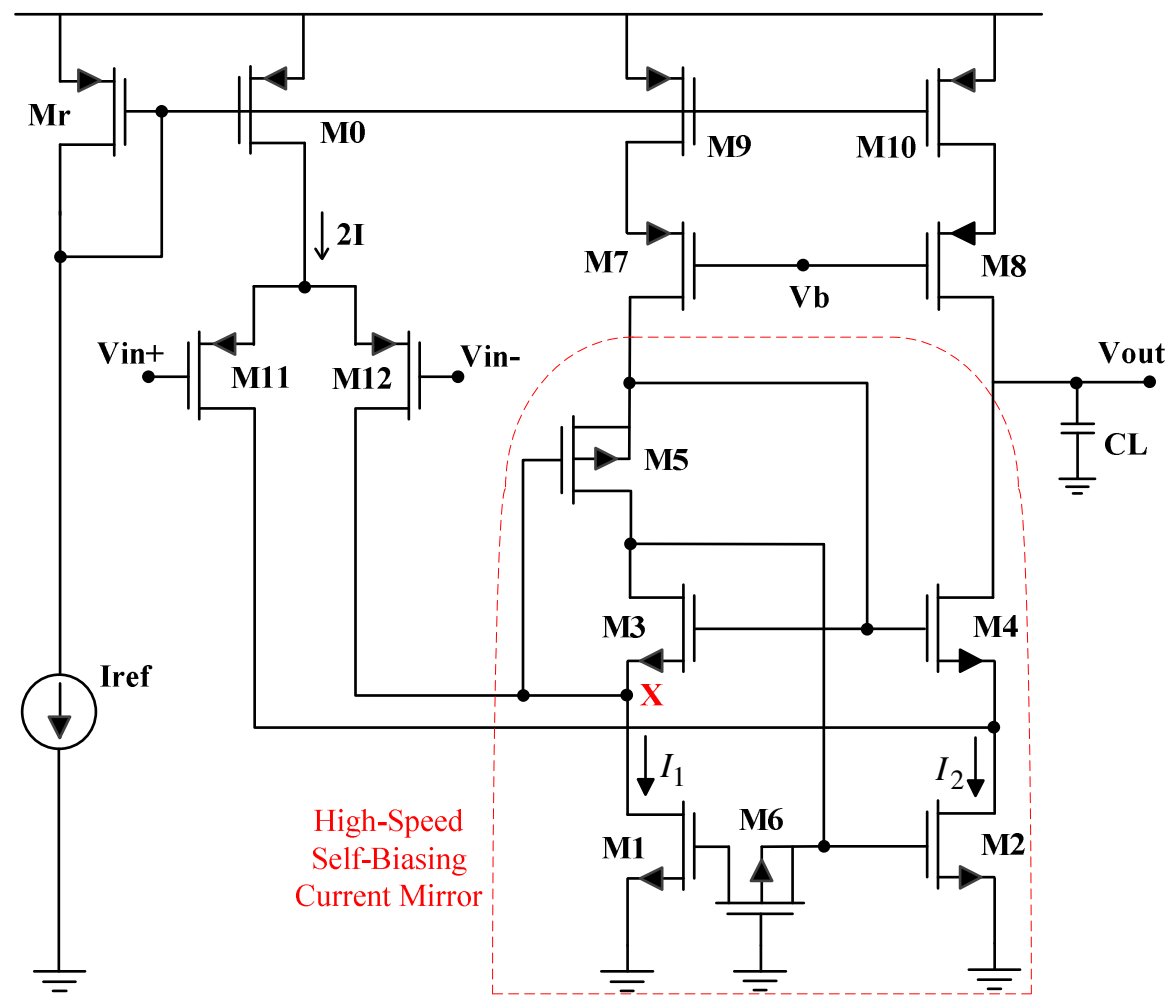

Figure 5. The proposed enhanced phase-margin folded cascode amplifier. 
The new pole and zero are described by (13) and (14), respectively.

$$
\begin{gathered}
\omega_{p 2}^{\prime}=-\frac{g m_{1}}{C_{g s 1}} \\
\omega_{z 2}=-\frac{1}{R_{6} C_{g s 1}}
\end{gathered}
$$

By choosing $R_{6}=1 / g m_{1}, \omega_{z 2}$ will be equal to $\omega_{p 2}^{\prime}$, thus the zero cancels the first non-dominant pole of the proposed amplifier and the new first non-dominant pole is determined by parasitic capacitor at folded node. Thus, the phase-margin of the proposed amplifier can be enhanced. Also, the high-speed CCM does not introduce additional poles and zeros. Therefore, the GBW of the proposed amplifier is not limited, which is some enhanced compared to that of the conventional FC.

\section{Simulation RESUltS}

The proposed current mirror (PCM) and proposed folded cascode (PFC) amplifier are simulated in $0.18 \mu \mathrm{m}$ CMOS technology with power supply voltage of $1.8 \mathrm{~V}$. The current gain error rate $\left(I_{\text {out }}-I_{\text {in }}\right)$ and the tolerable input voltage range of the proposed $\mathrm{CM}$ with comparison to the conventional CM is shown in Figs. 6a and 6b, respectively. The maximum current gain error rate of the proposed CM is $1.6 \%$ in the input current range of $12 \mu \mathrm{A}$ to $490 \mu \mathrm{A}$. Also in this range, the input voltage changes from $575 \mathrm{mV}$ to $1.6 \mathrm{~V}$. It is evident that both current error and input dynamic range are significantly improved. Namely, in the wide range of the input current/voltage variations, all transistors of the proposed $\mathrm{CM}$ will remain in the saturation region of the strong inversion to have a linear operation.

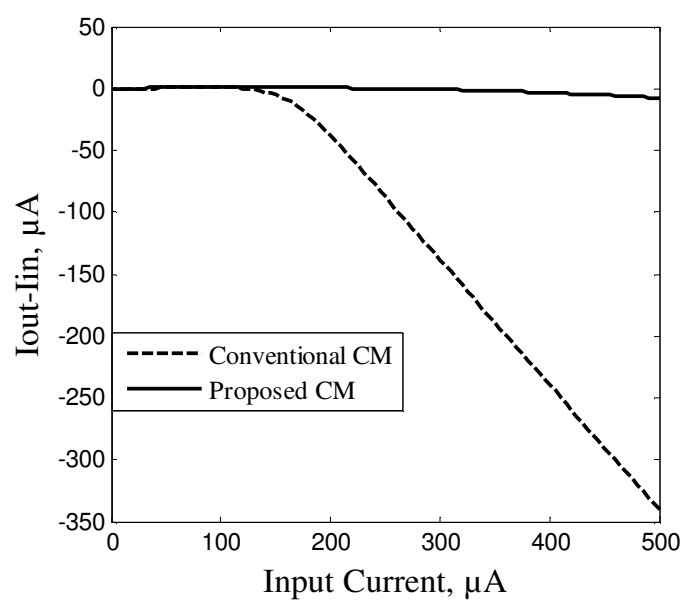

(a)

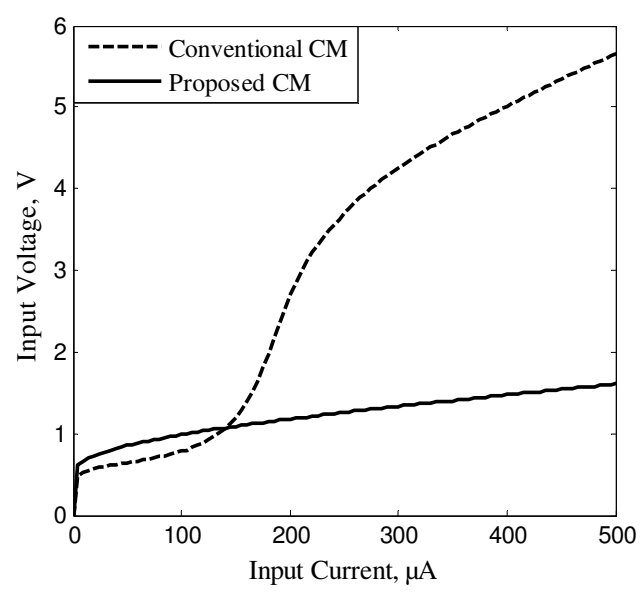

(b)

Figure 6. (a) The current gain error rate for proposed and conventional CMs, and (b) the Input voltage varations with respect to the input current for proposed and conventional CMs.

The frequency response of the proposed and conventional amplifiers is presented in Fig. 7a. This figure shows $222 \mathrm{MHz}$ bandwidth improvement of the proposed $\mathrm{CM}$ compared to the conventional CM. Also, the frequency response of the amplifiers is shown in Fig. 7b. For design of amplifiers, both the FC and PFC are loaded with $2.5 \mathrm{pF}$ capacitor. The size of transistor M6 is adjusted to cancel the first non-dominant pole and the phase-margin enhancement. It can be seen that the phase-margin is improved by $12^{\circ}$. Furthermore, the GBW is increased by $22.4 \mathrm{MHz}$ without sacrificing any performance. 


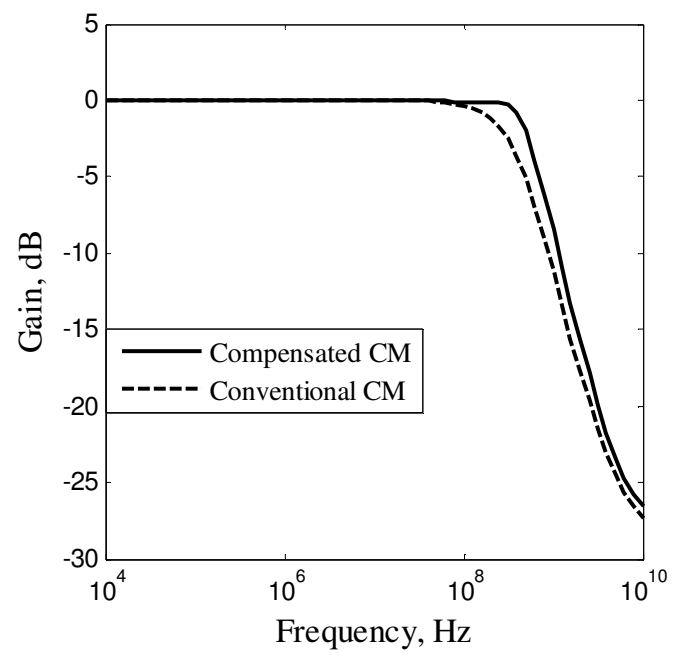

(a)

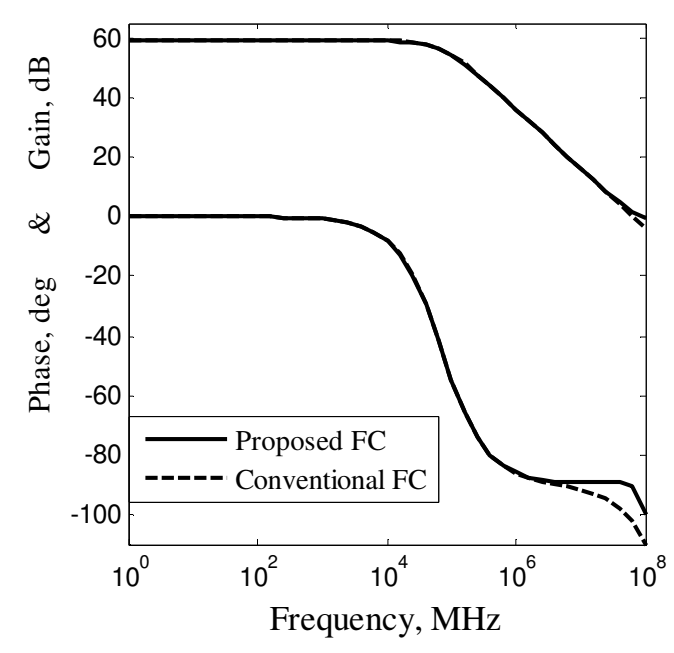

(b)

Figure 7. (a) Frequnecy resposne of the proposed and conventional FC amplifiers, and (b) frequnecy resposne of the compensated and conventional CMs.

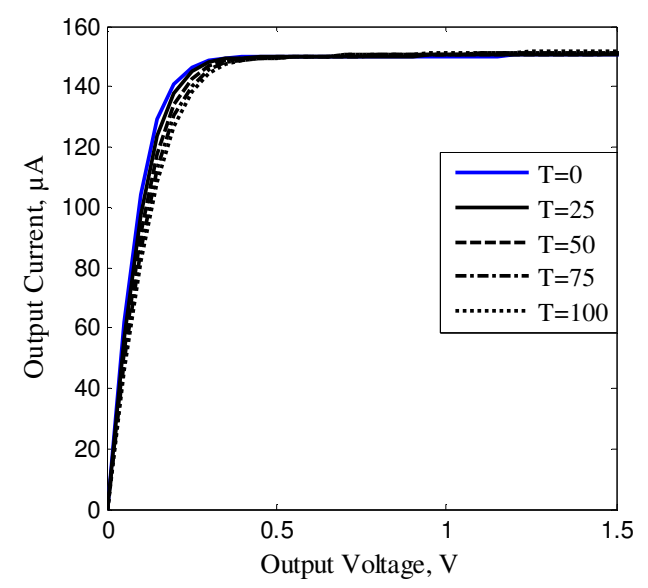

(a)

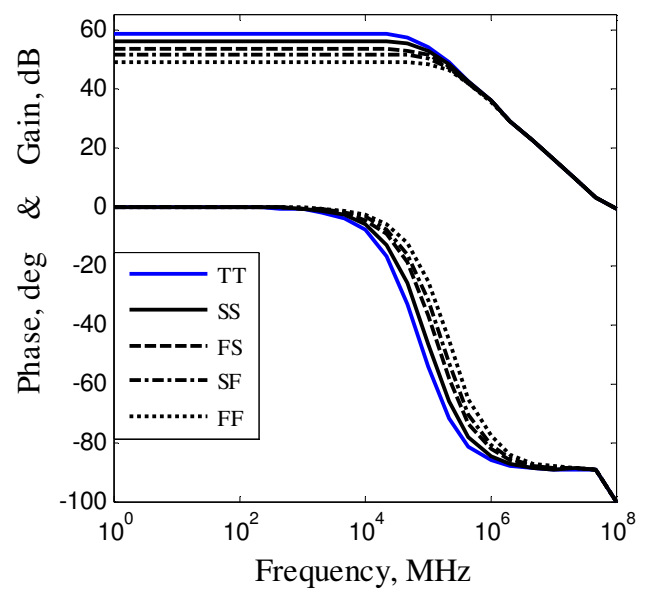

(b)

Figure 8. (a) Temperature variations of the proposed CM, and (b) corner analysis of the proposed FC amplifier.

The designed proposed circuits demonstrate relatively suitable response in different temperatures and process corners. Figs. $8 \mathrm{a}$ and $8 \mathrm{~b}$ show the effects of temperature dependent on output current of the proposed $\mathrm{CM}$ and corner processes on frequency response of the proposed FC, respectively. As shown, in FF corner, the bandwidth of the $\mathrm{PFC}$ is increased by $4 \%$ and the phase-margin by $2.8^{\circ}$ while the DC gain decreased by about $9 \mathrm{~dB}$. In SS corner, the bandwidth of the PFC is decreased by $3.5 \%$, the phase-margin by approximately $2.3^{\circ}$ and DC gain by $3.5 \mathrm{~dB}$. Table 1 summarizes the specifications of the PCM, PFC and recent works. Also, table 2 details the transistor sizes used in the simulation of the PCM and PFC circuits. To evaluate these works two figure of merits (FOM) for amplifiers and current mirrors can be defined by (15) and (16), respectively. 
Electrical and Electronics Engineering: An International Journal (ELELIJ) Vol 3, No 4, November 2014

$$
\begin{gathered}
F O M_{\text {OTA }}=\frac{(\text { DC gain })(\text { Unit gain frequency })(P . M)}{(\text { Power dissipation })} \\
F O M_{C M}=\frac{(\text { Input current swing })}{(\text { Maximum current } \text { gain error rate })(\text { Minimum input voltage })}
\end{gathered}
$$

Table 1. Specifications of the PCM and PFC in comparison with conventional circuits and to recent works.

\begin{tabular}{|c|c|c|c|c|c|c|c|}
\hline \multicolumn{4}{|c|}{ FC amplifiers } & \multicolumn{5}{|c|}{ Current mirrors } \\
\hline Specifications & FC & PFC & Specifications & CM & PCM & {$[3]$} & {$[3]$} \\
\hline Supply voltage $(\mathrm{V})$ & 1.8 & 1.8 & Supply voltage $(\mathrm{V})$ & 1.8 & 1.8 & 1.8 & 1.8 \\
\hline GBW $(\mathrm{MHz})$ & 63.7 & 86.1 & $\begin{array}{c}\text { Minimum input } \\
\text { voltage }(\mathrm{V})\end{array}$ & 0.35 & 0.575 & 0.9 & 0.575 \\
\hline DC gain $(\mathrm{dB})$ & 59 & 59 & $\begin{array}{c}\text { Minimum output } \\
\text { voltage }\end{array}$ & 0.35 & 0.35 & 0.35 & 0.35 \\
\hline $\begin{array}{c}\text { Power consumption } \\
(\mu \mathrm{W})\end{array}$ & 750 & 750 & $\begin{array}{c}\text { Input current swing } \\
(\mu \mathrm{A})\end{array}$ & 120 & 478 & 260 & 180 \\
\hline $\begin{array}{c}\text { Phase-margin } \\
(\text { degree })\end{array}$ & 74 & 86 & $\begin{array}{c}\text { Input voltage swing } \\
(\mathrm{V})\end{array}$ & 0.35 & 1.025 & 0.8 & 1 \\
\hline $\begin{array}{c}\text { Input voltage noise } \\
(1 \mathrm{kHz}(\mathrm{nV} / \mathrm{sqHz})\end{array}$ & 120 & 120 & $\begin{array}{c}\text { Maximum current } \\
\text { gain error rate }\end{array}$ & High & $1.6 \%$ & $3.1 \%$ & $2.8 \%$ \\
\hline $\begin{array}{c}\text { Estimated area } \\
\left(\mu \mathrm{m}^{2}\right)\end{array}$ & 5000 & 5000 & $\begin{array}{c}\text { Estimated area } \\
\left(\mu \mathrm{m}^{2}\right)\end{array}$ & 450 & 500 & 600 & 600 \\
\hline $\begin{array}{c}\text { FOM } \\
(\mathrm{dB} . \mathrm{MHz} . \mathrm{deg} / \mu \mathrm{W})\end{array}$ & 370 & 582 & FOM $(\mu \mathrm{A} / \mathrm{V} . \%)$ & Low & 520 & 93 & 111 \\
\hline
\end{tabular}

Table 2. Transistor sizes $(\mu \mathrm{m})$ of the PCM and PFC circuits.

\begin{tabular}{|c|c|c|}
\hline Device & Proposed CM & Proposed FC \\
\hline Mb & - & $50 / 1$ \\
\hline M0 & - & $158 / 1$ \\
\hline M1, M2 & $30 / 1$ & $30 / 1$ \\
\hline M3, M4 & $15 / 1$ & $15 / 1$ \\
\hline M5 & $54 / 1$ & $54 / 1$ \\
\hline M6 & $14 / 1$ & $14 / 1$ \\
\hline M7, M8 & - & $86 / 1$ \\
\hline M9, M10 & - & $81 / 1$ \\
\hline M11, M12 & - & $171 / 1$ \\
\hline
\end{tabular}

\section{Conclusions}

A new biased cascode current mirror with high input/output swing without frequency response degeneration has been presented in this paper. By using proposed $\mathrm{CM}$ in folded cascode amplifier, the phase margin of the FC amplifier is improved without bandwidth limitation. Simulation results in $0.18 \mu \mathrm{m}$ CMOS technology exhibits $478 \mu \mathrm{A}$ linear input/output current swing of the proposed CM and a phase-margin enhancement of $12^{\circ}$ for the proposed FC amplifier compared to the conventional circuits, respectively.

\section{REFERENCES}

[1] P. J. Crawley and G. W. Roberts, "High-swing MOS current mirror with arbitrarily high output resistance," Electronics Letters, vol. 28, pp. 361-363, 1992. 
[2] J. Ramirez-Angulo, "Current mirrors with low input and low output voltage requirements," in Circuits and Systems, 1994., Proceedings of the 37th Midwest Symposium on, 1994, pp. 107-110 vol.1.

[3] E. Bruun and P. Shah, "Dynamic range of low-voltage cascode current mirrors," in Circuits and Systems, 1995. ISCAS '95., 1995 IEEE International Symposium on, 1995, pp. 1328-1331 vol.2.

[4] L. Ying-Chuan, W. Hung-Yu, J. Yuan-Long, and H. Yu-Wei, "A CMOS Current Mirror with Enhanced Input Dynamic Range," in Innovative Computing Information and Control, 2008. ICICIC '08. 3rd International Conference on, 2008, pp. 571-571.

[5] M. Akbari and O. Hashemipour, "Enhancing transconductance of ultra-low-power two-stage folded cascade OTA," Electronics Letters, vol. 50, pp. 1514 - 1516, 2014.

[6] M. Akbari, S. Biabanifard, S. Asadi, and M. C. E. Yagoub, "Design and analysis of DC gain and transconductance boosted recycling folded cascode OTA," AEU - International Journal of Electronics and Communications, vol. 68, pp. 1047-1052, 2014.

[7] T. Voo and C. Toumazou, "High-speed current mirror resistive compensation technique," Electronics Letters, vol. 31, pp. 248-250, 1995.

[8] X. Zhao, H. Fang, and J. Xu, "Phase-margin enhancement technique for recycling folded cascode amplifier," Analog Integrated Circuits and Signal Processing, vol. 74, pp. 479-483, 2013/02/01 2013.

[9] A. Ramazani, S. Biabani, and G. Hadidi, "CMOS ring oscillator with combined delay stages," AEUInternational Journal of Electronics and Communications, vol. 68, pp. 515-519, 2014.

\section{Authors}

Meysam Akbari was born in Kermanshah, Iran in 1988. He received the B.S. and M.S. degrees in Electrical Engineering from Kermanshah Islamic Azad University in 2010 and Shahid Beheshti University in 2013, respectively. His research interests include low power and high speed data converter, low voltage analogue circuits and RF integrated circuits design. He is currently with Department of Electrical and Computer Engineering and Microelectronic Laboratory in Shahid Beheshti University, G. C., Tehran, Iran.

Masoud Nazari received his B.S degree in 2011 at K.N.Toosi University of Technology and M.S degree in 2013 at Shahid Beheshti University both in Electronics Engineering. He is currently with the Microelectronic Research Laboratory in Shahid Beheshti University, Tehran, Iran. His research interests include low power and high speed data converter, low voltage analog circuits and RF integrated circuits design.

Ardavan Javid received the B.S. and M.S. degrees in electrical engineering from K.N. Toosi university of technology (KNTU), Tehran, Iran, in 2011 and 2013, respectively. Currently, he is associate researcher in Integrated Circuits and Systems laboratory (ICAS) at KNTU. His research interests include the digital wireless communication and mixed-signal integrated circuit for bioelectronics and implantable biomedical devices.
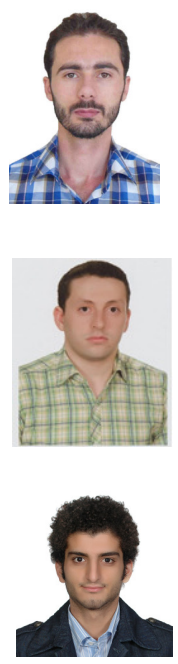